\title{
Estilos de aprendizaje e intención de uso de videos académicos de YouTube en el contexto universitario Chileno
}

\author{
Jonathan Tapia-Jara, Aurora Sánchez-Ortíz* y Cristian Vidal-Silva \\ Departamento de Administración, Facultad de Economía y Administración, Universidad Católica del Norte, Angamos \\ 0610, Antofagasta - Chile (e-mail: jonahtapia@outlook.com, asanchez@ucn.cl, cristian.vidal@ucn.cl) \\ * Autor a quien debe ser dirigida la correspondencia
}

Recibido May. 2, 2019; Aceptado Jun. 24, 2019; Versión final Ago. 1, 2019, Publicado Feb. 2020

\begin{abstract}
Resumen
Este trabajo analiza la relación entre los estilos de aprendizaje, motivación, satisfacción e intención de uso de videos de YouTube, un medio muy utilizado para la difusión y adquisición de información en todas las áreas, y en particular por los estudiantes universitarios. Se trabajó con una muestra de 235 estudiantes, $54 \%$ mujeres y $46 \%$ hombres, con un rango de edad predominante entre 18 y 22 años (50\%) (un $62 \%$ de mujeres y $50 \%$ de hombres en dicho rango, respectivamente) mediante un análisis descriptivo y de correlación. El análisis de datos muestra que más del $79 \%$ de los sujetos presentan una propensión al uso de videos de YouTube como apoyo en el proceso de aprendizaje. Los estudiantes con los estilos de aprendizaje convergente y asimilador presentan una mayor predisposición para usar estos videos, estudiantes que a su vez tienen una mayor motivación por utilizar videos académicos de YouTube. Estos hallazgos son relevantes para el análisis de la conveniencia del uso sistemático de videos en un contexto universitario.
\end{abstract}

Palabras clave: estilos de aprendizaje; YouTube; satisfacción; intención de uso; videos.

\section{Learning styles and intent of using YouTube academic videos in the Chilean university context}

\begin{abstract}
This work analyzes the relationship between learning styles, motivation, satisfaction and intention to use YouTube videos, a widely used medium for the dissemination and acquisition of information in all areas, in particular by university students. The study sample consisted of 235 students, $54 \%$ women and $46 \%$ men, with a predominant age range between 18 and 22 years (50\%) (62\% of women and $50 \%$ of men in that range, respectively) by means of a descriptive and correlation analysis. The data analysis shows that more than $79 \%$ of the students have a propensity to use YouTube videos to support the learning process. Students with convergent and assimilator learning styles present a greater predisposition to the use of those videos, students who also show a greater motivation for using YouTube academic videos. These findings are relevant for analyzing the convenience of the systematic use of videos in a university context.
\end{abstract}

Keywords: learning styles; YouTube; satisfaction; intention to use; videos. 


\section{INTRODUCCIÓN}

A lo largo de la historia, las formas de enseñanza y aprendizaje han ido evolucionando de acuerdo a los tiempos. Desde la antigua Grecia, en donde el saber quedaba reducido solo para algunos privilegiados, hasta las actuales formas de aprendizaje que surgen a partir de las nuevas tecnologías. Las Tecnologías de Información y Comunicaciones (TICs) en la actualidad están apoyando cada vez más el proceso de aprendizaje de los estudiantes facilitando la difusión de información y conocimiento. Estas tecnologías permiten superar brechas geográficas y socioeconómicas que limitaban la difusión y el acceso al conocimiento en el pasado (Hamid et al., 2015). Así, los videos académicos de YouTube (YouTube, 2019) se han transformado en un complemento importante a las clases presenciales en el contexto universitario ya que les permiten profundizar y aprender de formas diferentes.

La importancia que tienen los medios y las técnicas de enseñanza en la educación a distancia y, concretamente, el uso de los medios audiovisuales ha sido ampliamente destacado en la literatura actual (Brame, 2016; Perez et al., 2015; Akkoyunlu y Soylu, 2008; Choi y Johnson, 2005;). Tal y como resaltan Akkoyunlu y Soylu (2008), los videos son un recurso que proporciona diversas alternativas que pueden favorecer los procesos perceptivos y cognitivos durante el proceso de aprendizaje. La importancia de los videos se ha visto a nivel empírico, pero aún faltan estudios que indiquen como este uso se ve reflejado directamente en el aumento de satisfacción en el aprendizaje y motivación. Así mismo, Brame (2016), Perez et al. (2015), Akkoyunlu y Soylu (2008), y Choi y Johnson (2005) también han mencionado que los estilos de aprendizaje pueden producir diferencias en la satisfacción y motivación con los videos de apoyo al aprendizaje. La mayoría de las investigaciones coincide en que estos estilos son características internas predominantes que influyen en las formas en las que las personas perciben, recuerdan y piensan y que los estudiantes aprenden y procesan la información de diferente manera. Por ejemplo, algunos pueden preferir aprender a través de actividades en línea, mientras que otros pueden preferir aprender por métodos tradicionales (Ruiz, 2004).

Por tanto, es necesario conocer los diferentes estilos de aprendizaje, para identificar como el alumno se beneficiaría con el uso de videos académicos y potenciar su proceso de enseñanza-aprendizaje. Al conocer el estilo de aprendizaje de los alumnos también se les puede motivar mejor, despertar su interés en las materias y aumentar la satisfacción con el aprendizaje obtenido. Alonso Tapia (1997) comenta que existen conductas que contribuyen a motivar o desmotivar a los estudiantes, y que ciertas formas de aprendizaje tienen efectos distintos según el estilo de aprendizaje que se trate.

Por esta razón, para poder valorar adecuadamente los métodos que contribuyen la motivación de los alumnos, es preciso conocer previamente cuáles de sus características influyen en que estén más o menos motivados por aprender. Con respecto a la intención de uso de videos académicos, es necesario conocer qué estilo de aprendizaje se beneficia más con los videos on-line. Ya que, según Ross y Schulz (1999), puede que este medio de aprendizaje no sea el más adecuada para todos los alumnos, y que algunos alumnos se ven más favorecidos que otros. Para Salinas et al. (2008), la satisfacción del estudiante es el eje central de todos los procesos de aprendizaje ya que la satisfacción puede interceder positivamente o negativamente en la forma que los estudiantes aprenden y así disminuir las tasas de fracaso del estudiante (Pérez et al., 2015). El principal objetivo de este artículo es evaluar la relación entre los estilos de aprendizaje de los estudiantes universitarios en Chile, las variables de motivación, satisfacción e intención de uso de los videos de YouTube como parte del proceso de aprendizaje de dichos estudiantes.

\section{OTROS ANTECEDENTES}

Se presentan los constructos estilos de aprendizaje, satisfacción, motivación e intención de uso en el ámbito teórico, así como también algunas diferentes relaciones entre estos constructos en la literatura.

\section{Estilos de aprendizaje}

Tal y como lo señalan Ferrer (2012) y Schunk (2012), el estudio del aprendizaje refleja el deseo de las personas por entenderse a sí mismos y dar soluciones al proceso de aprendizaje. Alonso y Gallego (2007) consideran que los estilos de aprendizaje sirven como indicadores estables de como los alumnos perciben interacciones y responden a sus ambientes de aprendizaje. La tabla 1 resume los principales estilos de aprendizaje en la literatura. Hall y Moseley (2005) encuentran que los instrumentos más confiables de modelos de estilos de aprendizaje son los de Kolb $(1984 ; 2015)$, Honey y Mumford (1986) y Herrmann (1991). Este estudio utiliza el modelo de estilos de aprendizaje de Kolb $(1984 ; 2015)$ porque presenta ventajas para la evaluación de estudiantes a nivel universitario con respecto al uso de material visual (Kolb, 2015; Dillie y Mezack, 1991; Díaz y Cartnal, 1999; Miller, 2005; Liegle y Janicki, 2006). 
Tabla 1: Principales estilos de aprendizaje.

\begin{tabular}{|l|l|l|}
\hline Modelo & Autor & Tipos de estilo \\
\hline Estilo de Aprendizaje de Kolb & Kolb (1984; 2015) & Convergente, divergente, asimilador, acomodador \\
\hline Tipos de aprendizaje & $\begin{array}{l}\text { Honey y Mumford } \\
(1986)\end{array}$ & Activo, teórico, pragmático, reflexivo \\
\hline Estilo de aprendizaje & $\begin{array}{l}\text { Felder y Silverman } \\
(1988)\end{array}$ & Sensoriales, Visuales, Activos, Secuenciales \\
\hline $\begin{array}{l}\text { Programación Neurolingüística de } \\
\text { Bandler y Grinder }\end{array}$ & $\begin{array}{l}\text { Bandler y Grinder } \\
(1988)\end{array}$ & visual-auditivo-kinestésico \\
\hline $\begin{array}{l}\text { Cuadrantes cerebrales del } \\
\text { modelo de Ned Herrmann }\end{array}$ & Herrmann (1991) & Lógico, abstracto, secuencial, emocional \\
\hline Estilo de aprendizaje cognitivo & Gregorc (2001) & $\begin{array}{l}\text { Concreto-secuencial, abstracto-secuencial, } \\
\text { concreto-al azar, abstracto-al azar }\end{array}$ \\
\hline
\end{tabular}

\section{Modelo de aprendizaje de Kolb}

Kolb $(1984 ; 2015)$ indica que, a través de la búsqueda de experiencias, las personas programan su manera de aprender en las dimensiones del ciclo de aprendizaje. Los cuatro tipos de aprendizajes identificados por Kolb $(1984$; 2015) son: i) observación reflexiva, ii) conceptualización abstracta, iii) experimentación activa, y iv) experiencia concreta. Kolb $(1984 ; 2015)$ define dimensiones, las cuales en yuxtaposición forman un plano cartesiano en el que se clasifican los estilos de aprendizaje. La figura 1 ilustra dicho plano junto con las posiciones relativa de los estilos de aprendizaje junto con la caracterización de cada uno de los cuadrantes del plano los cuales se detallan en la tabla 2.

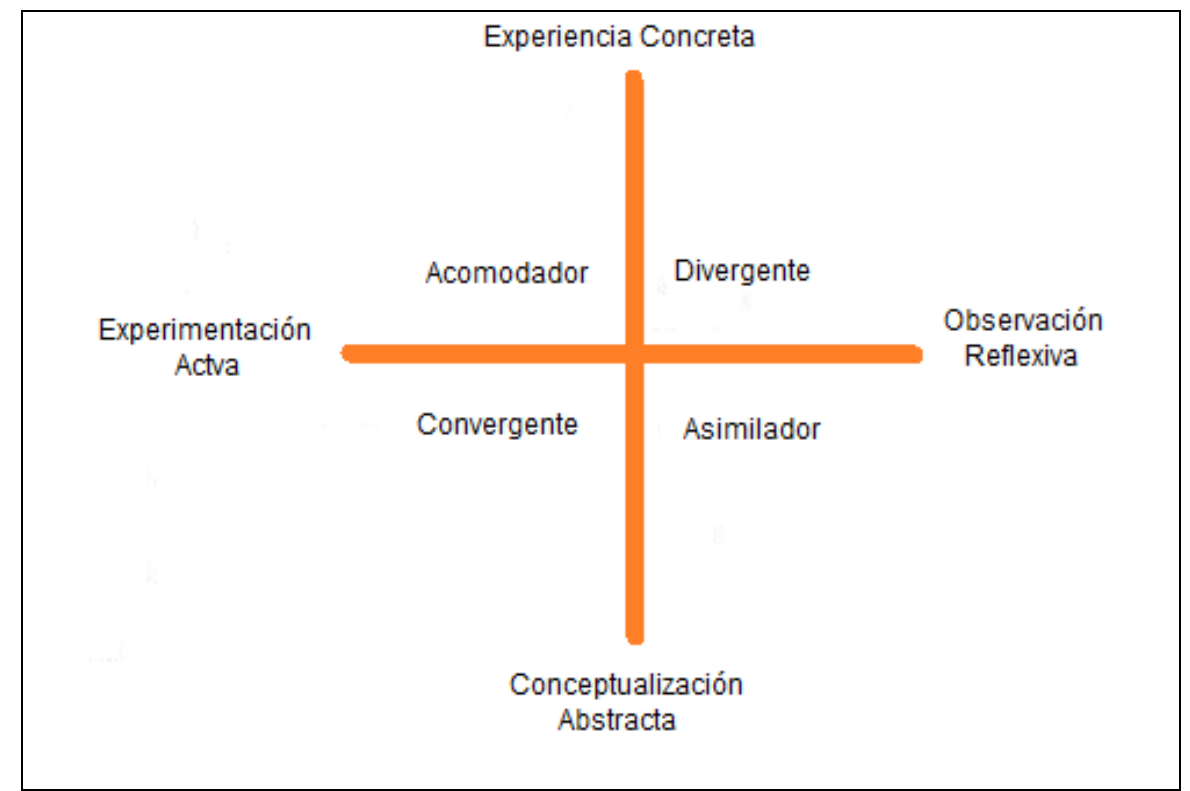

Fig. 1. Representación del Modelo de los estilos de aprendizaje de Kolb (Kolb, 1984; Kolb, 2015).

Tabla 2: Caracterización de estilos de aprendizaje de Kolb (Kolb, 1984; Kolb, 2015).

\begin{tabular}{|l|l|}
\hline \multicolumn{1}{|c|}{ Estilo } & \multicolumn{1}{c|}{ Características } \\
\hline Convergente & $\begin{array}{l}\text { Se caracterizan por ser buenos en tomar decisiones, aplicaciones prácticas, y en } \\
\text { resolución de problemas. Trabajan mejor donde hay solo una solución correcta ante } \\
\text { una situación }\end{array}$ \\
\hline Divergente & $\begin{array}{l}\text { Tienen habilidades imaginativas, ven situaciones concretas desde distintas } \\
\text { perspectivas. Son observadores y buenos en la lluvia de ideas. }\end{array}$ \\
\hline Asimilador & $\begin{array}{l}\text { Son inductivos, son capaces de crear modelos teóricos. Se enfocan menos en } \\
\text { personas y más en ideas y conceptos abstractos }\end{array}$ \\
\hline Acomodador & $\begin{array}{l}\text { Son intuitivos, aprenden con experiencia y buscan tomar riesgos. Son flexibles y } \\
\text { trabajan con otras personas. }\end{array}$ \\
\hline
\end{tabular}


El test de David Kolb (Kolb, 1984; Kolb, 2015) permite indicar los estilos de aprendizaje a través de la percepción y aplica 12 preguntas las que se responden en una escala de 1 a 4, donde 1 es la que más se aleja a la personalidad del individuo y 4 a la que más se acerca la personalidad. Esta prueba entrega un puntaje que permite posicionar en el diagrama de estilos de acuerdo a los valores para los ejes $\mathrm{X}$ e $\mathrm{Y}(\mathrm{X}=$ Experimentación Activa - Observación Reflexiva, e Y = Conceptualización Abstracta - Experiencia Concreta).

\section{Satisfacción y Motivación}

Según el diccionario Oxford (2016), la satisfacción es un sentimiento de bienestar o placer que se tiene cuando se ha colmado un deseo o cubierto una necesidad. Para Salinas et al. (2008), la satisfacción del estudiante es el eje central de todos los procesos que se llevan a cabo en las universidades pues su principal función sustantiva es la docencia centrada en él. Pérez et al. (2010) mencionan que algunos de los aspectos que se asocian a la satisfacción en la enseñanza son: i) La organización de las asignaturas (objetivos, contenidos), ii) El profesorado (capacidad, dominio), iii) La motivación (expectativas, dedicación), iv) Los recursos externos (infraestructura, biblioteca), v) La utilidad (personal, profesional). En la medida en que los sujetos tengan una actitud positiva respecto a estas cuestiones, podremos decir que están satisfechos.

La motivación se considera como un aspecto de enorme relevancia en el área educativa, por cuanto orienta las acciones que conduce lo que las personar realizan y hacía qué objetivos se dirige (Naranjo, 2009). Es así que, la motivación debe ser entendida como la trama que sostiene el desarrollo de aquella actividad que son significativas para la persona y en las que esta toma parte (Ajello, 2003). En términos de la educación, la motivación debe ser considerada como la disposición positiva para aprender y continuar haciéndolo de una forma autónoma.

\section{METODOLOGÍA}

Esta investigación es de tipo no- experimental por lo cual no se manipulan deliberadamente las variables en el estudio ya que se basan en las percepciones al momento de tomar la encuesta. El estudio propone un modelo que considera las variables motivación, satisfacción e intención de uso de videos académicos de YouTube según su relacionan con los estilos de aprendizaje. La Figura 2 presenta el modelo propuesto de investigación, que considera la relación que existe entre los constructos de estilos de aprendizaje y las variables satisfacción, motivación e intención de uso de videos académicos. En base al modelo propuesto y a la revisión de la literatura es posible proponer las siguientes hipótesis:

Hipótesis 1 (H1): El estilo de aprendizaje acomodador es el que presenta mayor motivación en el uso de videos académicos de YouTube. Los estilos de aprendizaje han sido estudiados por diversos autores, en donde se demuestra que la mayor motivación la presenta el estilo acomodador. Borracci (2015) estableció que los estudiantes con estilo acomodador se demoraron más tiempo en egresar, pero no abandonaron la carrera.

Hipótesis 2 (H2): La Motivación en el uso de videos académicos de YouTube esta positivamente relacionada con la satisfacción en su uso. La relación entre motivación y satisfacción ha sido estudiada por diversos autores en su gran mayoría esta relación ha sido positiva. Cortes (2015) estableció que las personas se sienten satisfechas cuando se les reconoce su esfuerzo, siendo esto un factor de motivación.

Hipótesis $3(\mathrm{H} 3)$ : El estilo de aprendizaje Convergente es el que presenta mayor satisfacción con el uso de videos académicos de YouTube. Los estilos de aprendizaje han sido estudiados por diversos autores, en donde se demuestra que la mayor satisfacción la presenta el estilo convergente.

Borracci (2015) estableció que los estudiantes con estilo convergente se sienten más satisfechos que los otros estilos.

Hipótesis $4(\mathrm{H} 4)$ : La intención de uso de videos académicos de YouTube está positivamente relacionada con la motivación al uso. La relación entre intención de uso y motivación ha sido estudiada por diversos autores, en su gran mayoría esta relación es positiva. Montero (2016) estableció que el usuario tendrá la intención de usar un sitio web, si es que esté sitio tiene factores motivadores como un aspecto agradable.

Hipótesis 5 (H5): La Intención de uso de videos académicos de YouTube está positivamente relacionada con la Satisfacción. La relación entre intención de uso y satisfacción ha sido estudiada por diversos autores, en su gran mayoría esta relación ha sido positiva. Montero (2016) estableció que la intención de usar de un sitio web está influenciada por la satisfacción del usuario.

\section{Población, muestra e instrumentos de medición}

La población del estudio son jóvenes universitarios chilenos que utilizan videos académicos de YouTube como parte de sus procesos de aprendizaje. Según el cenco del año 2017, Antofagasta está habitada por 361.873 personas, de las cuales, 130.274 (36\%) ingresaron a la educación superior, siendo esta la población utilizada 
para el estudio. Se calculo una muestra ajustada de estudiantes con el uso de la ecuación 1; en la cual, $\mathrm{N}$ es el tamaño de la población total, o sea 130.274 según CENSO 2017 (CENSO, 2019), e representa el margen de error ( $6 \%$ en este caso), $p$ es la proporción de individuos que posee las características del estudio $(0,5)$, y $z$ es la puntuación z de 1,65 determinada mediante el $90 \%$ del nivel de confianza deseado. Así, se estableció en 227 personas con un nivel de confianza del $95 \%$ y un error de 6, 5\%. La muestra final incluye a estudiantes universitarios que fueron contactados en las universidades del norte de Chile entre los meses de agosto y diciembre del 2018. Como resultado se recolectaron un total de 235 encuestas válidas, de estudiantes que tenían una edad igual o superior a 16 años.

$$
\text { Tamaño de la muestra }=\frac{\frac{z^{2} \times p \times(1-p)}{e^{2}}}{1+\left(\frac{z^{2} \times p \times(1-p)}{e^{2} \times N}\right)}
$$

Para efectos de recolección de datos, se utilizaron instrumentos de medición de los constructos estilo de aprendizaje, satisfacción, desempeño percibido, motivación e intención de manera presencial citando a grupos que respondían una encuesta online construida especialmente en Google Form (GoogleForms, 2019). Esta investigación utilizó 4 instrumentos que permitieron la recolección de datos y la medición de los constructos en el modelo (ver tabla 3). Estos instrumentos, que corresponden a un conjunto de preguntas cuyas respuestas se representan en una escala de Likert de 5 puntos, fueron adaptados a la realidad chilena y al lenguaje español.

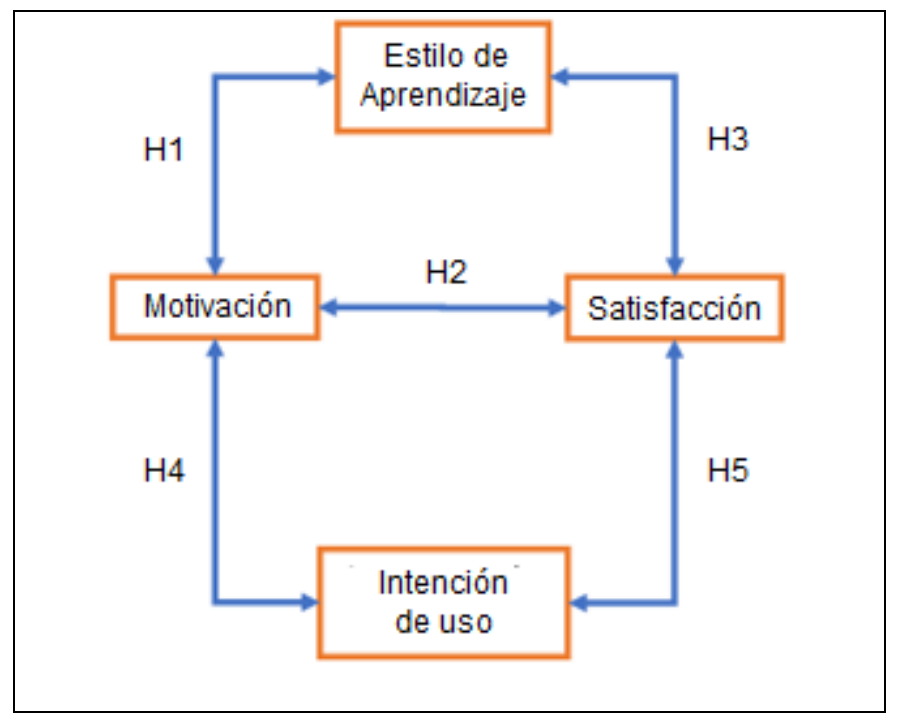

Fig. 2. Modelo propuesto de investigación.

Tabla 3: Instrumentos de medición de las variables.

\begin{tabular}{|l|l|}
\hline \multicolumn{1}{|c|}{ Constructo } & \multicolumn{1}{c|}{ Autor } \\
\hline Estilos de aprendizaje & Kolb (1984) \\
\hline Motivación & Tuan, Chin, y Shieh. (2005) \\
\hline Satisfacción & Belanche, Casaló, \& Guinalíu, (2012) \\
\hline Intención de uso & Ramírez y Álfaro (2012) \\
\hline
\end{tabular}

\section{ANÁLISIS DE DATOS Y RESULTADOS}

Para este trabajo, se lograron recopilar 235 encuestas aplicadas a estudiantes universitarios de la región de Antofagasta las cuales el $54 \%$ correspondió al género femenino, y $46 \%$ al género masculino. Las edades predominantes de las personas encuestadas, con un $50 \%$, se ubicó entre los 18 y 22 años, siendo para las mujeres el mayor porcentaje el de "entre 18 y 22 años" (62\%), y para los hombres el "entre 23 y 26 años" $(50 \%)$. Cabe señalar que dichos estudiantes pertenecen en alto grado a universidades tradicionales $(77 \%)$ mientras el resto pertenece a otras entidades de educación superior (23\%). Este estudio se realizó durante los últimos 5 meses de 2018 (agosto a diciembre), a través de la plataforma de formularios de Google. 
Los estilos de aprendizaje predominantes del estudio son el de convergente y asimilador con $34 \%$ y $33 \%$ respectivamente. El estilo de aprendizaje menos representado en la muestra es el de acomodador con 14\%. Para ambos géneros los estilos predominantes son Convergente y Asimilador. Respecto a los estilos de aprendizaje por área de estudios, se logró distinguir que el estilo mayoritario fue convergente en las áreas de economía y administración, humanidades e ingeniería. El estilo asimilador fue mayor en las áreas de ciencias, arquitectura, economía y administración, y humanidades. Cabe señalar que este trabajo no presenta resultados respecto a los contextos específicos de los encuestados tales como centro de educación superior, carrera, o asignatura relacionada.

El análisis demográfico de la variable intención de uso indica que más del $79 \%$ de los estudiantes están de acuerdo o muy de acuerdo en que usaran los videos de YouTube en el futuro en las asignaturas de su carrera. La variable Motivación muestra que un $73 \%$ de los estudiantes piensa que aprender es importante porque con lo aprendido se pueden resolver problemas, un $75 \%$ de los estudiantes está en total acuerdo sobre lo importante de los videos es tener la oportunidad de satisfacer la curiosidad al aprender, y un $58 \%$ de los estudiantes está muy de acuerdo en utilizar los videos académicos de YouTube porque estos no ejercen ningún tipo de presión sobre ellos. La variable de Satisfacción muestra que más de un $80 \%$ de los estudiantes tomaría la decisión de utilizar los videos académicos de YouTube para aprender y más del $80 \%$ de los estudiantes se encuentra satisfecho con la experiencia de utilizar los videos académicos de YouTube. El análisis de los datos para evaluar las hipótesis presenta los siguientes resultados, con respecto a cad hipotesis.

\section{Resultados por hipótesis}

H1: El estilo de aprendizaje acomodador es el que presenta mayor motivación en el uso de videos académicos de YouTube. Se utilizó el test Chi-Square del análisis de contingencia para cruzar las variables motivación y estilo de aprendizaje, y como se aprecia en la tabla 4, no hay diferencias significativas en los estilos de aprendizaje para esta variable. Al compara las medias de los estilos, se encontró que el valor más alto se da en el estilo de aprendizaje divergente y no el acomodador (ver tabla 5). Por lo anterior, esta hipótesis no es soportada por los datos y no es posible rechazar la hipótesis nula.

Tabla 4: Prueba Chi-Cuadrado para Estilo de Aprendizaje y Motivación con el uso de videos académicos de YouTube.

\begin{tabular}{|c|c|c|c|}
\hline & Valor & $\mathrm{gl}$ & Significación Asintotica (Bilateral) \\
\hline Pearson Chi-Cuadrado & $15,596^{\mathrm{a}}$ & 12 & ,210 \\
\hline Tasa de Probabilidad & 16,814 & 12 & 157 \\
\hline $\begin{array}{l}\text { Línea por Línea } \\
\text { Asociación }\end{array}$ & 2,759 & 1 & 097 \\
\hline $\mathrm{N}$ de Casos Válidos & 235 & & \\
\hline
\end{tabular}

Tabla 5: Medias para variable Motivación por Estilo de aprendizaje

\begin{tabular}{|l|r|r|r|r|}
\cline { 2 - 5 } \multicolumn{1}{c|}{} & \multicolumn{4}{c|}{ Estilo } \\
\cline { 2 - 5 } \multicolumn{1}{c|}{} & Divergente & Asimilador & Acomodador & Covergente \\
\hline Media & 4,58 & 4,3 & 4,34 & 4,3 \\
\hline
\end{tabular}

H2: La Motivación en el uso de videos académicos de YouTube esta positivamente relacionada con la satisfacción en su uso. Se utilizó el análisis de correlación de Pearson entre las variables motivación con el uso de videos académicos de YouTube y satisfacción con el uso de estos videos. El resultado muestra que existe una muy alta correlación, .669, que es significativa en el nivel 0,01 (bilateral). Este resultado permite soportar la hipótesis presentada y por tanto rechazar la hipótesis nula (ver tabla 6).

H3: El estilo de aprendizaje convergente es el que presenta mayor satisfacción con el uso de videos académicos de YouTube. Se utilizó el test Chi-Square del análisis de contingencia para cruzar las variables estilos de aprendizaje y satisfacción con el uso de videos académicos de YouTube y tal como se aprecia en la tabla 7, no hay diferencias significativas en los estilos de aprendizaje para esta variable. Al compara las medias de los estilos, se encontró que el valor más alto se da en el estilo divergente y no en el convergente (ver tabla 8). Este resultado no permite rechazar la Hipótesis nula. 
H4: La intención de uso de videos académicos de YouTube está positivamente relacionada con la motivación al uso. Se utilizó el análisis de correlación de Pearson para analizar la relación que existe entre las variables intención de uso de videos académicos de YouTube y motivación. El resultado muestra que existe una muy alta correlación del .645 la que es significativa en el nivel 0,01 (bilateral). Este resultado permite soportar la hipótesis presentada y rechazar la hipótesis nula (ver tabla 9 ).

Tabla 6: Correlación de Pearson entre Motivación y Satisfacción con el uso de videos académicos de YouTube.

\begin{tabular}{|c|c|c|c|}
\hline & & Motivación & Satisfacción \\
\hline \multirow{3}{*}{ Motivación } & Correlación de Pearson & 1 & ,669" \\
\hline & Sig. (bilateral) & &, 000 \\
\hline & N & 235 & 235 \\
\hline \multirow{3}{*}{ Satisfacción } & Correlación de Pearson & $669^{* *}$ & 1 \\
\hline & Sig. (bilateral) & ,000 & \\
\hline & $N$ & 235 & 235 \\
\hline
\end{tabular}

Tabla 7: Tabla de contingencia de Estilo y Motivación con el uso de videos académicos de YouTube.

\begin{tabular}{|l|r|r|rr|}
\hline & \multicolumn{1}{|c|}{ Valor } & \multicolumn{1}{|c|}{ gl } & \multicolumn{2}{|c|}{$\begin{array}{c}\text { Significación Asintotica } \\
\text { (Bilateral) }\end{array}$} \\
\hline Pearson Chi-Cuadrado & $13,478^{\mathrm{a}}$ & 12 &, 335 \\
\hline Tasa de Probabilidad & 16,639 & 12 &, 164 \\
\hline $\begin{array}{l}\text { Línea por Línea } \\
\text { Asociación }\end{array}$ & 5,419 & 1 &, 020 \\
\hline N de Casos Válidos & 235 & & & \\
\hline
\end{tabular}

Tabla 8: Medias para Variable Satisfacción por estilo de aprendizaje

\begin{tabular}{|l|r|r|r|r|}
\cline { 2 - 5 } \multicolumn{1}{c|}{} & \multicolumn{4}{c|}{ Estilo } \\
\cline { 2 - 5 } \multicolumn{1}{c|}{} & Divergente & Asimilador & Acomodador & Covergente \\
\hline Media & 4,52 & 4,25 & 4,34 & 4,08 \\
\hline
\end{tabular}

Tabla 9: Correlación de Pearson entre Intención de Uso y Motivación con el uso de videos académicos de YouTube.

\begin{tabular}{|l|l|c|c|}
\hline \multicolumn{2}{|c|}{} & Intención de Uso & Motivación \\
\hline \multirow{3}{*}{$\begin{array}{l}\text { Intención de } \\
\text { Uso }\end{array}$} & Correlación de Pearson & 1 &, $645^{* *}$ \\
\cline { 2 - 4 } & Sig. (bilateral) & &, 000 \\
\cline { 2 - 4 } & $\mathrm{N}$ & 235 & 235 \\
\hline \multirow{3}{*}{ Motivación } & Correlación de Pearson &, $645^{* *}$ & 1 \\
\cline { 2 - 4 } & Sig. (bilateral) &, 000 & \\
\cline { 2 - 4 } & $\mathrm{N}$ & 235 & 235 \\
\hline
\end{tabular}

H5: La Intención de uso de videos académicos de YouTube está positivamente relacionada con la satisfacción. El análisis de correlación de Pearson permitió analizar la relación que existe entre las variables intención de uso de videos académicos de YouTube y satisfacción. El resultado que se presenta en la tabla 10 indica que existe una muy alta correlación del .650 significativa en el nivel 0,01 (bilateral). Este resultado permite soportar la hipótesis presentada y rechazar la hipótesis nula. 


\section{DISCUSIÓN}

Los resultados obtenidos en esta investigación indican el rol relevante de los estilos de aprendizaje en la satisfacción e intención de uso de los videos de YouTube como complemento de los procesos de aprendizaje. Se pone en evidencia también que existe una alta predisposición por parte de todos los estudiantes a nivel universitario a utilizar estos medios audiovisuales como apoyo en su proceso de formación. El estudio permitió evidenciar que existe una mayor predisposición al uso de estos videos por parte de los estudiantes con los estilos de aprendizaje Convergente y Asimilador. Así mismo, los estudiantes con el estilo Convergente y divergente tienen una mayor motivación al utilizar videos académicos de YouTube.

Tabla 10: Análisis de correlación de Pearson entre Intención de Uso y Satisfacción con el uso de videos de YouTube.

\begin{tabular}{|l|l|c|c|}
\hline \multicolumn{2}{|l|}{} & Intención de Uso & Satisfacción \\
\hline \multirow{4}{*}{ Intención de Uso } & Correlación de Pearson & 1 &, $650^{* *}$ \\
\cline { 2 - 4 } & Sig. (bilateral) & &, 000 \\
\cline { 2 - 4 } & $\mathrm{N}$ & 235 & 235 \\
\hline \multirow{4}{*}{ Satisfacción } & Correlación de Pearson &, $650^{* *}$ & 1 \\
\cline { 2 - 4 } & Sig. (bilateral) &, 000 & \\
\cline { 2 - 4 } & $\mathrm{N}$ & 235 & 235 \\
\hline
\end{tabular}

El análisis de los datos mostró una relación muy significativa entre las variables motivación y satisfacción. Esta relación es muy importante y se podría explicar ya que los estudiantes al poder aprender sin ninguna presión estimulan su pensamiento y al mismo tiempo pueden resolver sus problemas sobre la materia del curso logrando sentirse más motivados al usar videos académicos de YouTube, y logrando así, una experiencia satisfactoria al aprender. Por otra parte, uno de los resultados obtenidos que es de relevancia del estudio es la alta correlación que existe entre satisfacción y motivación y entre satisfacción e intención de uso. Ambos resultados indican que sin duda el uso de videos de YouTube es fundamental en el trabajo académico pero este uso está muy relacionado con cuanto motivan estos videos (calidad y entretención) y esto entonces se podría reflejar en la intención de uso de estos videos para mejor el desempeño académico en el futuro. Futuras investigaciones se deben centrar en evaluar si existe diferencia entre el desempeño académico percibido por los estudiantes, sus estilos de aprendizaje y las calificaciones reales. Así también es importante estudiar si existen diferencias en las calificaciones de exámenes entre los estudiantes que utilizan videos académicos de YouTube y los estudiantes que no los utilizan.

En este artículo se ha discutido la relevancia de los estilos de aprendizaje en la motivación, satisfacción e intención de uso de los videos académicos de YouTube. Los hallazgos de esta investigación sugieren que el aprendizaje depende de variados factores y el estilo de aprendizaje es un factor muy relevante en el tema. Estas características son únicas de cada estudiante en el contexto universitario y deben ser consideradas al momento de diseñar asignaturas con material audiovisual como videos de YouTube. En este sentido, Balakrishnan y Gan (2016) indican que los alumnos con diferentes estilos de aprendizaje abordan el aprendizaje de manera diferente. También estos autores destacan la importancia del análisis de los factores que impulsan a los estudiantes con diferentes estilos de aprendizaje a usar las tecnologías y redes sociales en el proceso de enseñanza aprendizaje de manera más efectiva. El estudio permitió determinar que existía una relación muy significativa entre las variables motivación, satisfacción e intención de uso de los videos de YouTube como parte del proceso de aprendizaje de los estudiantes y sin duda este conocimiento aportará en el gran desafío que está enfrentando la educación hoy en día como es la virtualización de la enseñanza y como hacer un mejor uso de la tecnología para apoyar estos procesos. En la misma línea, Shoufan (2019) estudió en qué medida los videos educativos en YouTube admiten las características cognitivas formuladas en la teoría cognitiva del aprendizaje multimedia y como estas características apoyan el aprendizaje de los estudiantes. Sus resultados muestran que las características cognitivas significativas son: entrenamiento previo, modalidad, contigüidad espacial y realización. A partir de esto, el autor indica la necesidad de realizar investigaciones adicionales para identificar y especificar características cognitivas adicionales en los videos educativos hacia una comprensión más profunda del valor cognitivo del video.

En algunas áreas disciplinarios el tiempo de estudio es limitado en el campus por lo que se necesitan recursos de aprendizaje flexibles, tecnológicamente apropiados, y accesibles de forma remota; áreas en las que YouTube sería una herramienta adecuada. Por ejemplo, Johnston (2018) realizó un experimento en el ámbito de la enfermería creando una colección de videos que se publicaron en el Canal de Ciencias Biológicas de YouTube con la intención de mejorar las competencias de estudiantes en biociencia clínica el que fue muy exitoso con más de 310,000 visitas y 1,5 millones de minutos de visualización y más de 5000 suscriptores. 
Los resultados del estudio sobrepasaron las expectativas del autor evidenciando su utilidad y sugiriendo que los videos enriquecen perceptiblemente la experiencia y el rendimiento de los estudiantes en la biociencia. Las hipótesis planteadas en el modelo fueron soportadas en su totalidad y el análisis también permitió ver que algunos estilos de aprendizaje están más motivados para el uso de estos videos que otros estilos. Este estudio servirá para apoyar el desarrollo de cursos a nivel universitario que se centren más en el uso de herramientas digitales como son los videos de YouTube para hacer más eficiente el proceso de enseñanza-aprendizaje. Algunas importantes limitaciones del estudio son que se realiza solo con estudiantes de universidades del norte de Chile y que el instrumento de estilos de aprendizaje de Kolb fue complejo y requirió explicación detallada para que fuese comprendido por los estudiantes.

Es importante señalar que, aun cuando YouTube es una rica fuente de videos de carácter educativo y académico, no todos los videos en YouTube que usan para con dicho fin son necesariamente de buen nivel. Así, los resultados de este estudio apuntan principalmente al uso de videos de YouTube con fines académicos, pero no necesariamente al uso directo de videos académicos creados y respaldados por docentes y profesionales de la academia, lo cual se puede considerar como una limitante respecto a los resultados obtenidos.

\section{CONCLUSIONES}

Según los resultados obtenidos de este trabajo, se plantean las siguientes conclusiones: 1. YouTube representa un estilo de red social para compartir videos cuyo impacto en el la educación, y en generación y creación de conocimiento gracias a videos académicos es de un alto valor para las nuevas generaciones de estudiantes; 2. Las entidades de educación superior deben considerar la oportunidad de difusión y acceso al conocimiento mediante YouTube para adaptar las formas tradicionales de educación con la inclusión de esta plataforma cuyo acceso es aún libre, así como acceder a material de otras casas de estudios de connotado prestigio internacional; 3. Aun cuando la existencia y masificación de YouTube sea una realidad para las nuevas generaciones, igual es necesario la presencia de los estudiantes para preservar la interacción entre estudiantes y trabajo en equipo, así como para la generación de debate para el desarrollo de competencias blandas.

\section{REFERENCIAS}

Akkoyunlu, B. y M. Y. Soylu, A Study of Student's Perceptions in a Blended Learning Environment Based on Different Learning Styles, Journal of Educational Technology \& Society, 11:1, 183 - 193 (2008)

Alonso Tapia, J., Motivar para el aprendizaje: Teorías y estrategias, ISBN: 84-236-4346-8, EDEBE, Barcelona, España (1997)

Alonso, C. y D. Gallego, Los Estilos de Aprendizaje: Procedimientos de Diagnóstico y Mejora, ISBN: 978-84-271-1914-7, Editorial Mensajero, Edición 7, Bilbao, España, Enero (2007)

Balakrishnan, V. y C. L. Gan, Students' Learning Styles and their Effects on the Use of Social Media Technology for Learning, Telematics and Informatics, 33(3), 808 - 821 (2016)

Bandler, R. y J. Grinder, Use su Cabeza para Cariar, Seminario de Programación Neurolingüística (PNL), Santiago, Chile (1988)

Borracci, R. A., Estilos de Aprendizaje de Kolb en Estudiantes de Medicina, Medicina, 75(4), 73 - 80 (2015)

Brame, C. J., Effective Educational Videos: Principles and Guidelines for Maximizing Student Learning from Video Content, doi: 10.1187/cbe.16-03-0125, CBE Life Sciences Education, 15(4), Octubre (2016)

CENSO, Resultados CENSO 2017, (en línea), http://resultados.censo2017.cl/. Acceso: 23 de Julio (2019)

Choi, H. J. y S. D. Johnson, The Effect of Context-Based Video Instruction on Learning and Motivation in Online Courses, doi: 10.1207/s15389286ajde1904_3, American Journal of Distance Education, 19(4), 215 - 227, Diciembre (2005)

Cortes, V. L., La motivación Laboral y su Incidencia en el Desempeño Organizacional en Empresas Copelec, Tesis de grado, Facultad de Ciencias Empresariales, Universidad del Bio-Bio, Chillán, Chile (2015)

Diaz, D. P. y R. B. Cartnal, Students' Learning Styles in Two Classes: Online Distance Learning and Equivalent onCampus, doi: 10.1080/87567559909595802, College Teaching, 47(4), 130 - 135 (1999)

Dillie, B. y M. Mezack, Identifying predictors of high-risk among community college telecourse students, The American Journal of Distance Education, (5:1), 24 - 35 (1991)

Felder, R. M. y L. L. Silverman, Learning and Teaching Styles in Engineering Education, Engineering Education, 78, 674 -681, Enero (1988)

Ferrer, A., Analizar el Contexto para Obtener el Máximo Beneficio de la Evaluación, ISSN: 0210-5934, Revista de pedagogía, Bordón, 64(2), 13 - 28 (2012)

GoogleForms, Formularios de Google, (en línea), https://www.google.com/forms/about/. Acceso: 20 de Marzo (2019) 
Gregorc, A., An Adult's Guide to Style: contains the Gregorc Style Delineator, Gregorc Associates Inc., Columbia, USA (2001)

Hall, E. y D. Moseley, Is there a Role for Learning Styles in Personalized Education and Training?, doi: 10.1080/02601370500134933, International Journal of Lifelong Education, 24 (3), 243 - 255, Agosto (2006)

Hamid, S., J. Waycott, S. Kurnia y S. Chang, Understanding Students' Perceptions on the Benefits of Online Social Networking Use for Teaching and Learning, doi: 10.1016/j.iheduc.2015.02.004, The Internet and Higher Education, Elsevier, 26, 1 - 9, Marzo (2015)

Herrmann, N., The Creative Brain, Brain Books, North Carolina, Lake Lure, USA, Diciembre (1991)

Hernández Ruiz, L., La Importancia de los Estilos de Aprendizaje en la Enseñanza de Inglés como Lengua Extranjera, ISSN-e 1139-3637, Revista de Estudios Literarios, № 27 (2004)

Honey, P. y A. Mumford, A., The Manual of Learning Styles, ISBN: 095084440 3, Ardingly House, Maidenhead, Berkshire, Inglaterra (1986)

Johnston, A. N., M. J. Barton, G. A. Williams-Pritchard y M. Todorovic, YouTube for Millennial Nursing Students; Using Internet Technology to Support Student Engagement with Bioscience, doi: 10.1016/j.nepr.2018.06.002, Nurse Education in Practice, 31, 151 - 155 (2018)

Kolb, D., Experiential Learning: Experiences as the source of Learning Development, Prentice Hall, Nueva York, USA (1984)

Kolb, D., Experiential Learning: Experiences as the source of Learning Development, Prentice Hall, 2da Edición, Nueva York, USA (2015)

Liegle, J. O. y T. N. Janicki, The effect of learning styles on navigation needs of Web-based learners, doi: 10.1016/j.chb.2004.03.024, Computers in Human Behavior, 22(5), 885 - 898 (2006)

Miller, M. L., Using Learning Styles to Evaluate Computer based Instruction, doi: 10.1016/j.chb.2004.02.011, Computers in Human Behavior, 21(2), 287 - 306 (2005)

Montero, Y. H., Factores del diseño web orientado a la satisfacción y no-frustración de uso, doi: 10.3989/redc.2006.v29.i2.291, Revista Española de Documentación Científica, 29(2), 239 - 257 (2006)

Perez, F., P. Martinez y M. Martinez, Satisfacción del estudiante universitario con la tutoría. Diseño y validación de un instrumento de medida, doi: 10.15581/004.29.81-101, Estudios sobre la Educación, Vol. 29, 81 - 101 (2015)

Ross, J. y R. Schulz, Can Computer-Aided Instruction Accommodate all Learners Equally?, doi: 10.1111/14678535.00087, British Journal of Educational Technology, 30(1), 5 - 24, Diciembre (1998)

Salinas, A., J. Morales y P. Martínez, Satisfacción del Estudiante y Calidad Universitaria: un Análisis Multidisciplinar en la unidad académica multidisciplinaria agronomía y ciencias de la universidad autónoma de Tamaulipas, México, issn: 1131 5245, Revista de Enseñanza Universitaria, 31, 39 - 55, Universidad de Sevilla, España (2008)

Shoufan, A., Estimating the Cognitive Value of YouTube's Educational Videos: A Learning Analytics Approach, doi: 10.1016/j.chb.2018.03.036, Computers in Human Behavior, 92, 450 - 458 (2019)

Schunk, D. H., Teorías del Aprendizaje, Prentice Hall Latinoamérica, México (2012)

YouTube, YouTube, (en línea), https://www.youtube.com. Acceso: 14 de Marzo (2019) 